\title{
Effect of Certain Compounds on the Pigmentation Characteristics of Some Bacterial Isolates
}

\author{
Mohammed Abdul Aleem Qureshi $^{1^{*}}$, Saravanan Sankaran ${ }^{2}$ and Sowrirajan Subbaiah ${ }^{3}$ \\ ${ }^{1}$ Environmental Engineering Department, College of Engineering, Imam Abdulrahman Bin \\ Faisal University, Dammam, Saudi Arabia \\ ${ }^{2}$ Department of Life Sciences, King Fahad University of Petroleum \& Minerals, Dhahran, \\ Kingdom of Saudi Arabia \\ ${ }^{3}$ Department of Chemistry, King Fahad University of Petroleum \& Minerals, Dhahran, \\ Kingdom of Saudi Arabia \\ *Corresponding author
}

\section{A B S T R A C T}

\section{Keywords}

Pigmentation Characteristics, pigmented colonies

\section{Article Info}

Accepted:

16 December 2017

Available Online:

10 January 2018
Microorganisms produce various pigments like carotenoids, melanin's, flavins, monascins, violacein and indigo. Carotenoids are a group of bioactive compounds and are responsible for bright yellow, orange, red pigments of various plants, microorganisms and animals and are widely distributed in nature. These pigments have an important function to act as protective agents against oxidative damage. Recently carotenoids have attracted greater attention due to the beneficial role on human health. As pigments have been extensively evaluated over the years for its antibiotic, antigenic and antitumor effects, special consideration was made towards ability of chromogenic bacteria to produce pigmentation under certain chemical conditions.

\section{Introduction}

Many of the pigments are derivatives of certain groups of chemicals such as carotenoids, phenazine or pyrollic dyes, azaquinones and anthocyanates

The microbial pigments are secondary metabolites, that is, they are compounds not found in all organisms. They are not derived from the normal blocks and metabolites of cells.

Some bacterial species produce and retain water insoluble pigments intracellularly, thus causing the colonies to become colored (pigmented)

Some species which form pigmented colonies are

Serratia marcescens - Red

Staphylococcus aureus - Gold

Chromobacterium Violaceum - Violet

Flectobacillus major - Pink

Some colonies produce pigments that are water soluble for instance, Pseudomonas 
aeruginosa forms a blue water-soluble pigment called pyocyanin. Some pigments are only sparingly water soluble and may precipitate in the medium, for example Pseudomonas chhororaphis forms a pigment called chlororaphin which accumulates in the form of green crystals around the colonies.

Certain water-soluble pigments are fluorescent i: e the agar medium around the colonies grows white or blue-green when exposed to ultraviolet light. For example, $P$. aeruginosa produce notonly, the non-fluorescent pigment pyocyanin but also fluorescent pigment pyoverdine

For a bacterial strain to exhibit its characteristic pigmentation special media incubation temperatures or other conditions may be required. For instance, Mycobacterium kansasii forms a characteristic yellow pigment (b-carotene) only when the colonies are exposed to light (Table 1).

Pigmented forms are common among airborne inocula because the pigments, protect against the rays of the visible and near UV light ranges. Thus, on straw and dust and in other locations that are exposed to light, the ability to form such pigments confers a selective advantage over non-pigmented forms, which are more easily inactivated by light.

This protection against photodynamic damage was demonstrated in a study of a normally orange-colored, carotenoid-containing strain of halophilic bacterium and its colorless mutant. The bactericidal effect of visible light occurred under aerobic conditions and thus was apparently due to photo-oxidation

In photo-oxidation, several cellular pigments (flavins, cytochromes) apparently acts as catalysts. The carotenoids, localized in the cytoplasmic membrane, protect the sensitive regions of the cell from the effects of photo- oxidation. Pigments produced by microorganisms are as follows

Pyocyanin is excreted by Pseudomonas aeruginosa

Iodine is excreted by Chromobacterium iodinum

Indigoidin is produced by several bacterium including Pseudomonas indigofera.

\section{Antibiotic nature of pigment prodigiosin}

Prodigiosin is the characteristic red pigment commonly produced by Serratia marcescens.

Kraft (1902) extracted the red prodigiosin from Bacillus prodiosus

Observations of antibiotic properties of pigmented cultures of Serratia marcescens were first reported in 1885, these early investigation (1949) described antagonistic effects both in-vitro and in-vivo of pigmented cultures against many bacterial and fungal species. Since that time the pigment has been evaluated for its antibiotic antigenic and antitumor effects

The antibiotic nature of prodigiosin and inspite of the importance many other antibiotics have served in current chemotherapy

The observations by Caujolle et al., (1967) of malformations induced by DMSO were similar in few cases to those induced by the whole pigment and two of the pigment fractions, malformations of beak (parrot beak in prodigiosin c-2 and a-3) and anopthalmia of the left eye (c-2 and a-3)

Special consideration should be declared towards the inability of prodigiosin to inhibit Staphylococcus aureus at a concentration of $12.9 \mu \mathrm{g}$ in $0.03 \mathrm{ml}$ DMSO, whereas EO4 
demonstrated a positive zonal inhibition. Efimenko et al., (1956) and Burgova et al., (1957) reported that they found a fraction that possessed antibiotic activity, which appears to be different in many physical properties from our fractions. Both stated that this antibiotic fraction showed a red coloration, however in the present study the only fraction that came close to this description was the c-2 fraction which proved to be totally worthless as an antimicrobial agent in-vitro and was highly toxigenic during embryogenesis. The f-4 fraction from the pigment prodigiosin has been shown to be least embryotoxic and to inhibit the growth of several species of bacteria.

None of the discs charged with pigment fraction in petroleum ether, chloroform, acetone, ethanol and methanol exhibited any growth inhibition for all organisms tested. Pseudomonas spp was used only in the sensitivity studies incorporating the solvent DMSO. From the result it can be seen that PE1, C-2 and A-3 in DMSO also failed to exhibit any in-vitro antimicrobial activity. Thus, eliminating these fractions as possible bactericidal substances, however prodigiosin, E-4 AND M-5 in DMSO showed definite growth inhibition for organisms tested including Pseudomonas. The comparable antibiotic properties between the whole pigment and E-4 fraction, least zonal inhibition occurred with M-5.

Treatment of soil with an aqueous, membrane filtered solution $(3,5 \mathrm{mg} / \mathrm{ml})$ of the salt causes the soil microorganisms to fluoresce when examined with light from a mercury arc light source. The magnesium salt of 1-anilino-8napthalene sulfonic acid (Mg-Ans Nutritional biochemical, develand, ohio) was reported to fluoresce upon binding to serum albumin (weber and Laurence, 1954).

Lepierre (1898) studied a fluorescent bacillus.
He found that the only common organic acid salts, which gave fluorescence when used with proper inorganic constituents, were citric, succinic. Oxy-glutanic and glutaric acid. He concluded that fluorescence depends, first upon on the dibasicity of the acid, second upon the presence of at least two ch2 groups in the molecule.

\section{Effect of certain compounds on pigmentation}

Jordan (1899) and Sullivan (1905) stated that sulphates and phosphates regardless of the base, are the only inorganic elements necessary for the production of fluorescence.

Media were prepared containing 0.2 percent asparagine, with varying amounts of dipotassium phosphate and sulphates of sodium, potassium and ammonium, and also sulphuric acid. Control media were also prepared from each of the above media with the addition of 0.01 percent anhydrous magnesium chloride. Very little fluorescence was produced in any of the media without magnesium.

In some cases, the media containing the larger amounts of the sulphates gave a slight production of pigment.it was thought that these might contain a small amount of magnesium as an impurity.

In studying the media made from the different sulphates, it was found that the greater fluorescence was produced by the sample containing the sulphates of alkalis, gave little or no fluorescence, but in every case where magnesium chloride was added a good fluorescence was produced.

In order to prove definitely that the alkali sulphates will not suffice to produce fluorescence, it was decided to prepare a sulphate absolutely free from magnesium. 
Ammonium hydroxide and sulphuric acid were mixed in proper proportion to form ammonium sulphate. A medium was made, using this pure sulphate with a pure sample of dipotassium phosphate and asparagine. The medium made with ammonium sulphate in place of magnesium sulphate will not produce fluorescence.

The media contained 0.2 percent asparagine, varying amounts of potassium phosphate from 0.0001 to 0.1 percent and ten different amounts (from 0.00001 to 0.5 percent) of the magnesium chloride or magnesium ammonium chloride. The media containing the chlorides free from sulphates, gave very little fluorescence. The samples of chlorides which contained the sulphate as impurity gave much more fluorescence especially when the larger amount of magnesium chloride was used. This indicates that the sulphate is necessary for pigment production.

Several sets of media were made with asparagine and magnesium sulphate, but no fluorescence was observed, when a phosphate was added, a good fluorescence was produced, it may be concluded, therefore, that in addition to organic constituent such as asparagine the medium must contain a phosphate, magnesium and sulphate.

In order to determine the amounts of different constituents which, when present in a medium, will produce the best fluorescence. Media were, therefore made with varying amounts and combinations of the three ingredients.

The fluorescence in the lower percentage of asparagine was about equally good.

The medium containing 0.3 percent was probably a little better. The pigment production was very good when the phosphate content was between 0.005 and 0.1 percent, and when the magnesium sulphate was between 0.001 and 0.2 percent. The best and quickest pigment production was in the media containing 0.3 percent asparagine, 0.05 percent dipotassium phosphate and 0.05 percent magnesium sulphate.

In the light of the above, the following medium gives as the most satisfactory results for the production of fluorescence.

Magnesium sulphate anhydrous - $0.5 \mathrm{~g}$

Dipotassium phosphate anhydrous $-0.5 \mathrm{~g}$

Asparagine - $3.0 \mathrm{~g}$

Distilled water - $1000 \mathrm{cc}$

\section{Pigmentation of halophilic bacteria}

Bacteria living in highly concentrated saline media have shown two physiological types with different levels of adaptation to high salinity, the moderate halophiles and the extreme halophiles (flanned, 1956, Larsen, 1962, Dundas, 1977).

Both groups are adapted to live in this type of environment. The first is quite heterogenous and includes bacteria that in general show a wide growth range with respect to salt, their optimum being usually around 1-2 $\mathrm{M} \mathrm{NaCl}$.

The moderate halophiles, which grow over a much wider range of salt concentration than the extreme halophiles, are not greatly different physiologically from fresh water or marine bacteria (Kushner, 1978).

The extreme halophiles, are by contrast, drastically modified. They all adapt their internal ion concentrations to the environment (Larsen, 1967) and their proteins have a strong acidic composition that makes their structures, salt dependent (Langi, 1974). In addition, the cell envelop is of acidic character and lacks peptidoglycan (Larsen, 1967). The salt range which permits growth, with few exceptions, has a lower limit around 2-3 $\mathrm{M} \mathrm{NaCl}$, and is 
optimal at 4-5 M, most or all can grow in saturated $\mathrm{NaCl}$ (Larsen, 1967; Dundas, 1977)

In nutrient rich batch cultures, the moderate halophiles show a faster growth rate than shown by the extreme halophiles, even at the higher $\mathrm{NaCl}$ concentrations preferred by the extreme types (Forsyth and Kushner, 1970, Matheson et al., 1976).

Competition among halophilic bacteria can be studied more easily, since the extreme halophiles are strongly pigmented due to red or pink carotenoids whereas the moderate halophiles, with the exception of the redpigmented Spirochaeta halophila (Greenberg and Canaleparola, 1976), are non-pigmented or show only brown or yellow pigments.

Pigmentation permits differentiation between colonies of the two groups and facilitates the use of mixed natural populations of the two groups of microorganisms

Temperature seems to be the dominant selective factor in the interval of 20-30 percent salt concentration, the moderate halophiles being favored by lower temperatures. This fact is not surprising considering the demonstrated thermophilism of extreme halophiles (Keradiopoulos and Halldorf, 1977). A fourth retinal-containing pigment in Halobacterium halobium cell membrane was examined by flash spectrophotometry.

The absorption maximum of this pigment was at about 480 NM. Flash light caused a photoreaction cycle. The photoreaction cycle involved at least two photo intermediates. The absorption maximum of the first one was at about $350 \mathrm{~nm}$ and that of the second was at around 530nm. The spectral properties of this pigment and the content of the cell correlate with the sensitivity of photorepellet response to the light around $480 \mathrm{~nm}$ and suggest a name phoborhodopsin for this new pigment

Wild type Halobacterium halobium cells are attracted to yellow red light (positive phototaxis) and repelled by near-UV/blue light (negative phototaxis). Retinal containing pigments function as the photoreceptors. Three rhodopsin like pigments are so far known in $H$. halobium membrane: Bacteriorhodopsin (br) Halorhodopsin (hr) and Sensory rhodopsin (sr)

$\mathrm{Br}$ (a lambda $\max =568 \mathrm{~nm}$ and hr (lambda $\max =478 \mathrm{~nm}$ ) and show the phototaxis comparable to that of wild type strains.

Mutant (oni) which lacks the photo attractant response but shows an only photo repellent response whose sensitivity maximum is at around $480 \mathrm{~nm}$.It indicates that there is an additional photosystem other than the photosystem whose sensitivity maximum is around $370 \mathrm{~nm}$. Flash spectrophotometric data suggested that there was another retinal containing pigment (fourth rhodopsin like pigment) in cell membrane of $H$. halobium.

\section{Role of tyrosine in pigmentation}

Many species of bacteria and fungi have been reported to produce block of brown pigments from tyrosine (Ivins and Holmes, 1981, Barnett et al., 1983, Whealer, 1983; Vickers and Yu, 1984; Muller, 1985 Sadasivan and Neyro, 1985; Yoshimoto et al., 1985) among them, melanin's have been extensively studies and are the best-known group of pigments that are synthesized from tyrosine.

Serratia marcescens produce a brown pigment when grown in minimal medium in the presence of tyrosine and high concentrations of copper (ii) ion.

The pigment was not related to the melanin pigments, but was similar to the pigment produced by the autoxidation and polymerization of 3,4-dihydroxy phenylacetate, which is synthesized in $S$. marcescens from tyrosine through the 3,4- 
dihydroxyacetate catabolic pathway. Melanin's have been divided in to three classes, depending on the substrate used for pigment synthesis. Eumelanins are synthesized from tyrosine alone. In addition, cysteine is needed for phaeomelanin formation.

Allomelanins are derived from compounds such as acetate, catechol or 1,8dihydroxynapthalene (Alport and Buloch, 1958; Wheeler, 1983).

Among bacteria eumelanins have been demonstrated in actinomycetes (Kuster, 1976) and bacillus cereus (Aaronson and Vickers, 1965). Phacomelanin is produced by some vibrio strains (Pomerantz and murthy, 1974; Ivins and Holmes, 1981)

Some S. marcescens strains are able to produce different pigments. The best known prodigiosin is a linear tripyrrole attach to the cytoplasmic membrane (Vinas et al., 1983; Parachuri and Harshey, 1987). Some strains of S. marcescens belonging to the biotype A 40 synthesise a rose coloured diffusible pigment pyrimine (Grimont and Grimont, 1984)

Tyrosinase is a copper containing enzyme which catalyzes the first reaction of the melanogenesis pathway.

Black or brown pigments other than the melanin's have also been reported in Pseudomonas aeruginosa, where the pigment is formed by homogenestic and excreted into the medium (Yabuuchi and Ahyama, 1972). Muller (1985) demonstrated that the excretion of the alpha-ketoacids of the aromatic amino acids in the Morganella-Proteus-Providencia group yielded blackish pigment in the presence of iron under alkaline conditions.

\section{Influence of media composition on pigment formation}

A mineral medium (gl) for $S$. marcescens was prepared. Tyrosine was added to gl to give a final concentration of $1 \mathrm{~g} / \mathrm{l}$ (glt medium). $\mathrm{CuSO}_{4} 5 \mathrm{H}_{2} \mathrm{O}$ was added to various concentrations (from $0.8 \mu \mathrm{m}$ to $8 \mathrm{~mm}$ ). The effect of initial ph was studied from ph 5 to 9 and the influence of cysteine at concentrations of $1,0.1,0.01,0.001$ and $0 \mathrm{mg} / \mathrm{ml}$ was also investigated. Flasks with $95 \mathrm{ml}$ of the medium were inoculated with $5 \mathrm{ml}$ of an overnight culture grown in glt. Growth was monitored by following the od600 of the culture in spectrophotometer. Bacteria were removed by centrifugation and pigments production was measured in the supernatant fraction (OD 430) (Barnett et al., 1983).

The main characteristics of the brown pigments produced by $S$. marcescens compared with those produced by the autoxidation of dl-dihydroxyphenyl alanine and 3,4-dihydroxyphenylacetate. The properties of the brown pigments are closer to the autoxidized 3,4-dihydroxyphenylacetate than the autoxidized dldihydroxyphenylalanine. The substrates of aromatic ring cleavage dioxygenases (catechol, 2.5-dihydroxy phenylactate, 3,4dihydroxyphenylacetate and 3,4dihydroxybenzoate) and dldihydroxyphenylalanine were able to produce brown pigments.

Many strains of $S$. marcescens have been reported to produce different pigments. The production of brown pigments by $S$. marcescens has been demonstrated. This occurs when tyrosine is added to the medium. Since copper (ii) is needed to obtain a good pigmentation and alkaline ph enhances it. Pigment enhancement resulting from copper addition happened at high copper concentrations and it is unlikely that a tyrosinase-type enzyme needs such high copper concentrations to be achieve.

Mutants unable to use tyrosine as sole energy and carbon source could only produce the 
pigment if the pathway was blocked after the formation of 3,4-dihydroxyphenyl acetate. Many gram negative heterotrophic rods producing a violet pigment insoluble in water have been isolated. The physiology of these bacteria gave rise to several studies which resulted in their being classified within the genus, Chromobacterium (Cruess-Gallaghan and Gorman, 1935; Gilman, 1953; Leifson, 1956; Sneath, 1956a, 1956b; Eltinge, 1957)

Presence of spores or capsules, andgram staining were investigated using the techniques described by buttiaux et al., (1969). Flagellation was demonstrated by Rhodes method but because of instantaneous lysis occur with these strains in a non-saline medium, it was need to modify the method slightly according to a pattern describe before (Gauthier et al., 1975).

Several media were used for studying the pigments. The best synthesis results have been obtained on solid Zobel's medium 2216 added with $1 \%$ soluble starch $(\mathrm{w} / \mathrm{v})$. The violet pigment was extracted with ethanol $(90 \%)$ from separated by centrifugation (6000rpm, $4^{\circ} \mathrm{c} 15 \mathrm{~min}$ ). Another pigment, straw yellow in color and insoluble in ethanol was extracted in the same way using petroleum ether. The pigments were studied by UV and visible spectrophotometry

The potential growth of the strains at various temperature, ph and salinities in zobells medium 2216, the bacteria appear to be psychrophilic and grow in a moderately saline medium (15 to $50 \%$ ) i.e. with a salt content comparable with that of sea water. In addition, they require a neutral to alkaline ph to multiply and can usually tolerate a high alkalinity. Their old cultures become strongly alkaline.
The determination of the Na+ requirements of these strains show that their inability to grow in fresh water media is chiefly connected with the absence of that ion, their optimum $\mathrm{Na}+$ concentration is about $0.4 \mathrm{M}$, all being more or less inhibited at concentrations higher than $0.6 \mathrm{M}$. Violet types are more sensitive to low $\mathrm{Na}+$ concentrations than Alteromonas species, since they cannot grow in the presence of less than $0.1 \mathrm{M} \mathrm{Na}+$.

Bacteria of the genus, Pseudomonas may, under controlled nutritional conditions excrete water soluble fluorescent pigments in to the culture media. The ability to excrete the pigments is variable and depends on the composition of the growth medium.

Although all compounds essential for growth will certainly affect the production of the pigments. Various media have been defined for the evaluation of this important characteristic. Some require special ingredients (which if analyzed will be low in iron) and others require tedious and laborious removal by solvent extraction of the iron normally containing most ingredients of media.

The use of chelating agents and solvent extraction as suggested by Paton 101 is not only tedious and laborious, but if conducted with utmost efficiency will render the media iron free a nutritional condition permitting no growth of Pseudomonas.

\section{Effect of iron on pigmentation}

The principle factor, in addition to those essential for growth, which must be controlled to insole the excretion of fluorescent pigment by the genus Pseudomonas is the concentration of iron in the growth medium. 
Table.1 Colony characterization of different pigmented isolates obtained from milk and air sample

\begin{tabular}{|c|c|c|c|c|}
\hline \multirow{2}{*}{ Colony } & \multicolumn{5}{|c|}{ colony } \\
\hline characteristics & A & B & C & D \\
\hline Size & $2 \mathrm{~mm}$ & $3 \mathrm{~mm}$ & pinpoint & 1mm \\
\hline Shape & circular & circular & circular & circular \\
\hline Color & yellow & orange & cream & pink \\
\hline Margin & entire & entire & entire & entire \\
\hline Elevation & concave & concave & flat & concave \\
\hline Surface & smooth & smooth & smooth & smooth \\
\hline Opacity & opaque & opaque & opaque & opaque \\
\hline Consistency & sticky & sticky & sticky & sticky \\
\hline Grams nature & positive & negative & positive & positive \\
\hline Morphology & Cocci & Short rods & Short rods & coccobacillary \\
\hline Motility & -- & -- & -- & - \\
\hline $\begin{array}{c}\text { Pigment } \\
\text { solubility in }\end{array}$ & & & & + \\
\hline water & ++ & ++ & + & \\
\hline
\end{tabular}

Table.2 Biochemical characterization of the isolated Pigmented bacteria

\begin{tabular}{|c|c|c|c|c|}
\hline pigmentation & amylase & catalase & gelatinase & urease \\
\hline yellow & - & - & ++ & - \\
\hline cream & ++ & ++ & ++ & ++ \\
\hline pink & - & - & - & ++ \\
\hline orange & ++ & - & ++ & + \\
\hline
\end{tabular}

Table.3 Ultraviolet spectrophotometric characterization of yellow pigmented isolate in presence of various compounds

\begin{tabular}{|c|c|c|}
\hline Compound used & $\begin{array}{c}\text { Maximum absorption } \\
\text { observed (nm) }\end{array}$ & Corresponding O.D \\
\hline phosphate & & 0.010 \\
\hline $0.1 \%$ & 264.9 & 0.042 \\
\hline $0.05 \%$ & 387.9 & \\
\hline sulphate & & \\
\hline $0.1 \%$ & \multicolumn{2}{|c|}{ No peak detected } \\
\hline $0.05 \%$ & \\
\hline tyrosine & & \\
\hline $0.1 \%$ & &
\end{tabular}


Table.4 Ultraviolet spectrophotometric characterization of cream pigmented isolate in presence of various compounds

\begin{tabular}{|c|c|c|}
\hline Compound used & Maximum absorption observed $(\mathbf{n m})$ & Corresponding O. D \\
\hline phosphate & 207.5 & 1.822 \\
\hline $0.1 \%$ & 209.5 & 1.939 \\
\hline $0.05 \%$ & 211.0 & \\
\hline Sulphate & 209.5 & 2.220 \\
\hline $0.1 \%$ & & 2.006 \\
\hline $0.05 \%$ & 210.0 & \\
\hline tyrosine & 209.0 & 1.990 \\
\hline $0.1 \%$ & & 1.884 \\
\hline $0.05 \%$ & & \\
\hline
\end{tabular}

Table.5 Ultraviolet spectrophotometric characterization of orange pigmented isolate in presence of various compounds

\begin{tabular}{|c|c|c|}
\hline Compound used & Maximum absorption observed $(\mathrm{nm})$ & Corresponding O.D \\
\hline phosphate & & \\
\hline $0.1 \%$ & ---- & \\
\hline $0.05 \%$ & 207.5 & 1.842 \\
\hline Sulphate & & \\
\hline $0.1 \%$ & 209.4 & 2.078 \\
\hline $0.05 \%$ & 208.5 & 1.958 \\
\hline tyrosine & & \\
\hline $0.1 \%$ & 208.6 & 1.947 \\
\hline $0.05 \%$ & 214.0 & 2.298 \\
\hline
\end{tabular}

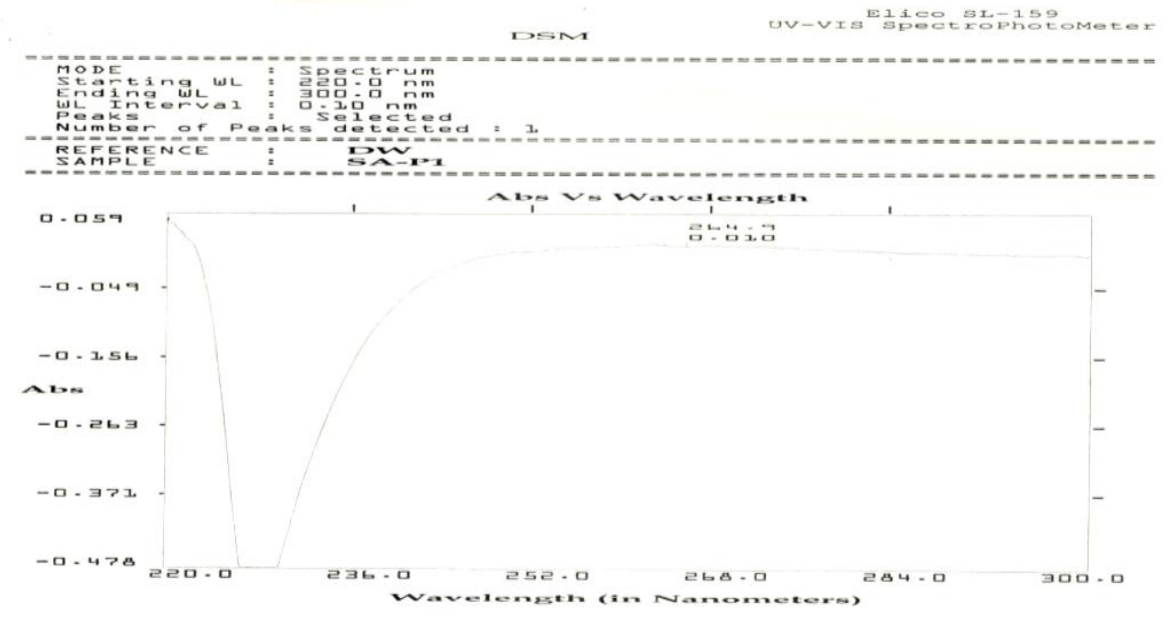



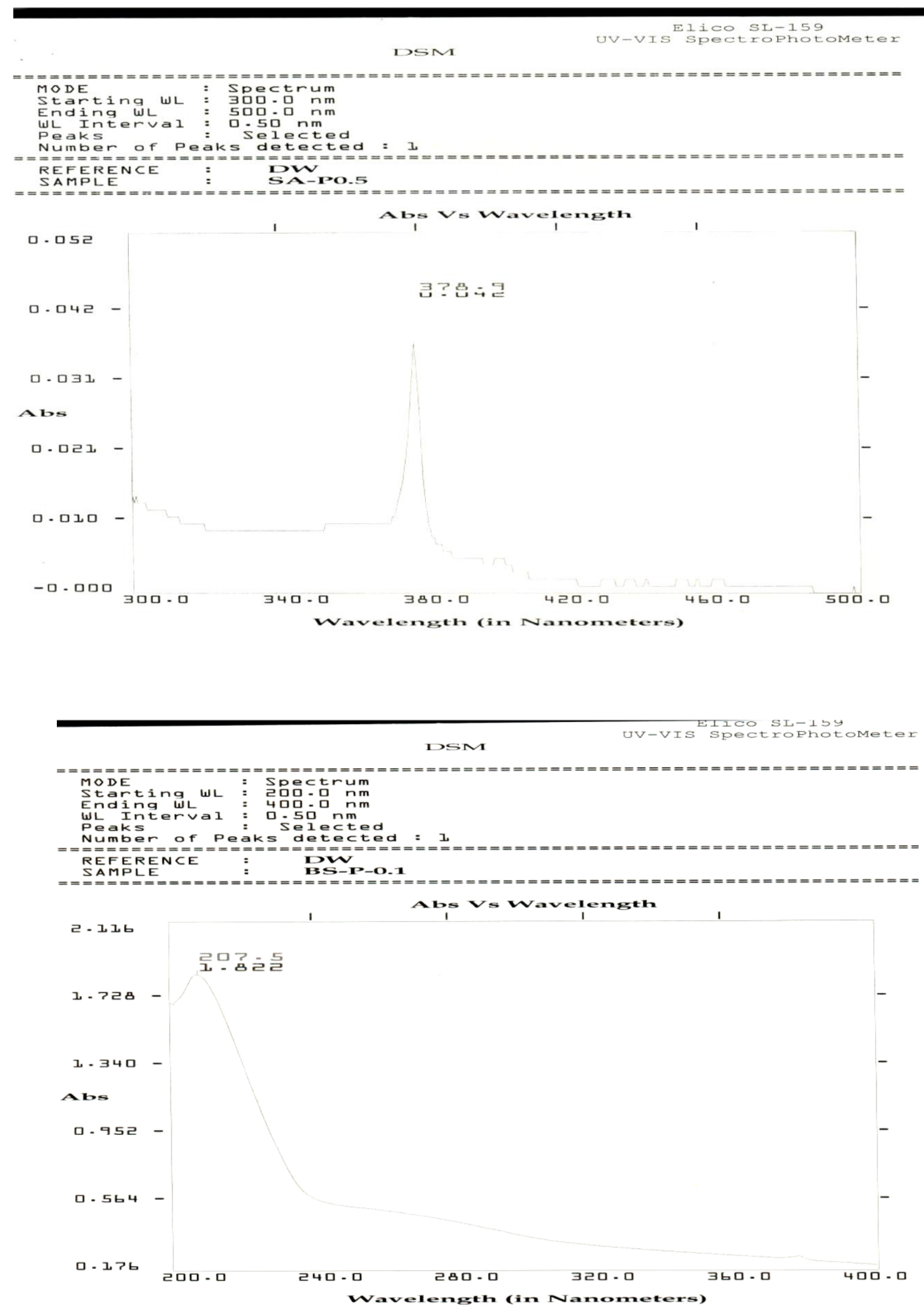

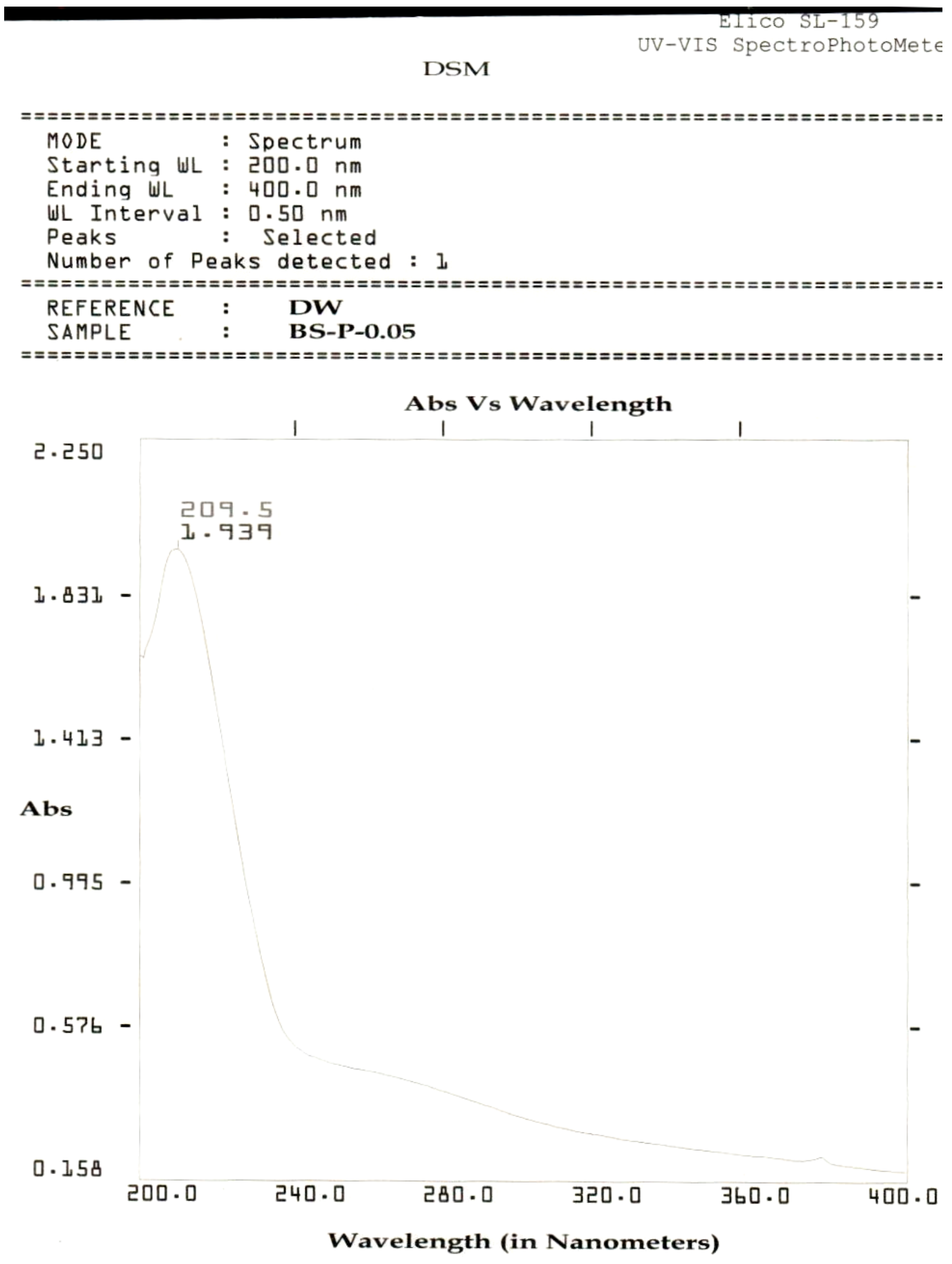


\section{DSM}
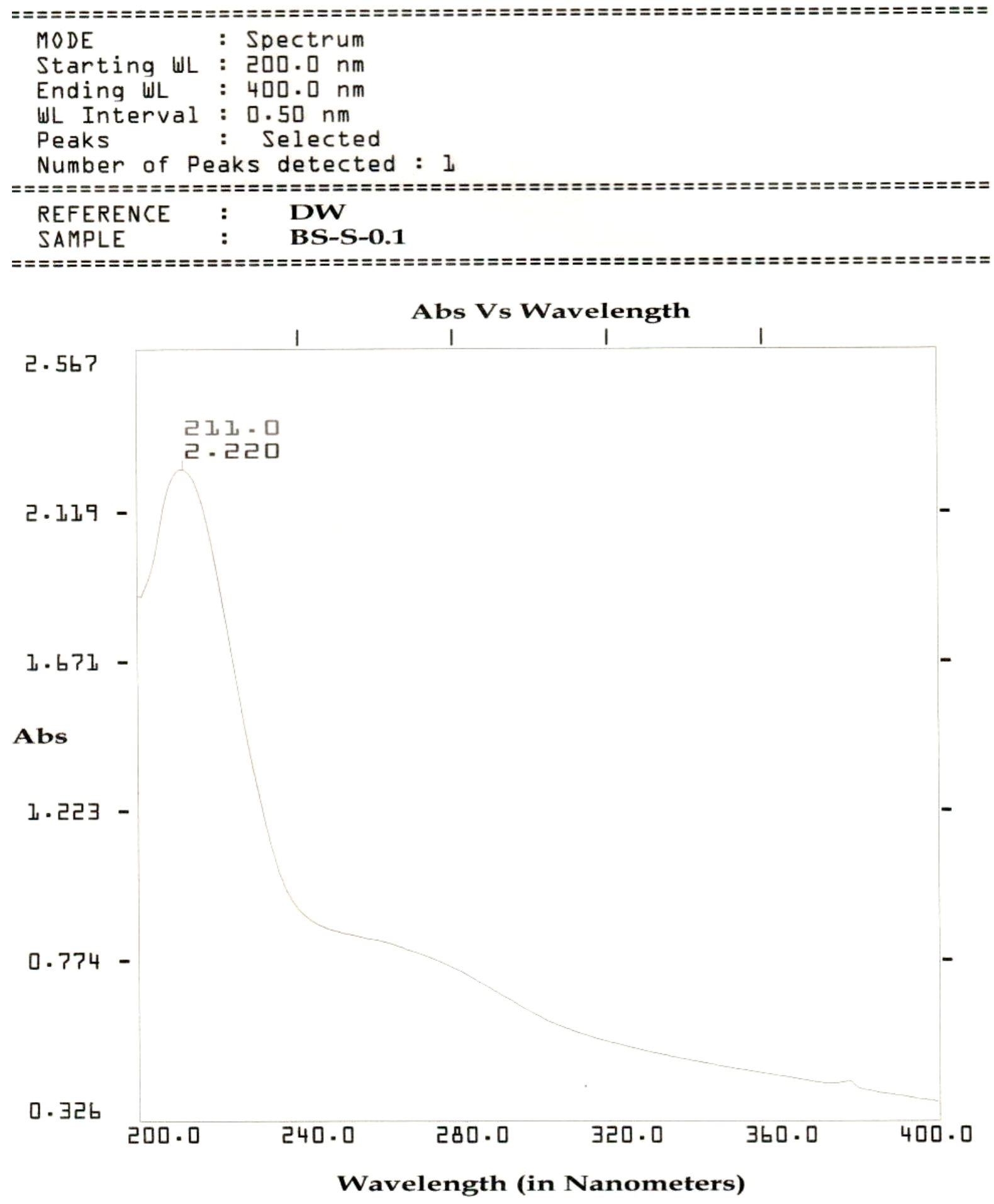

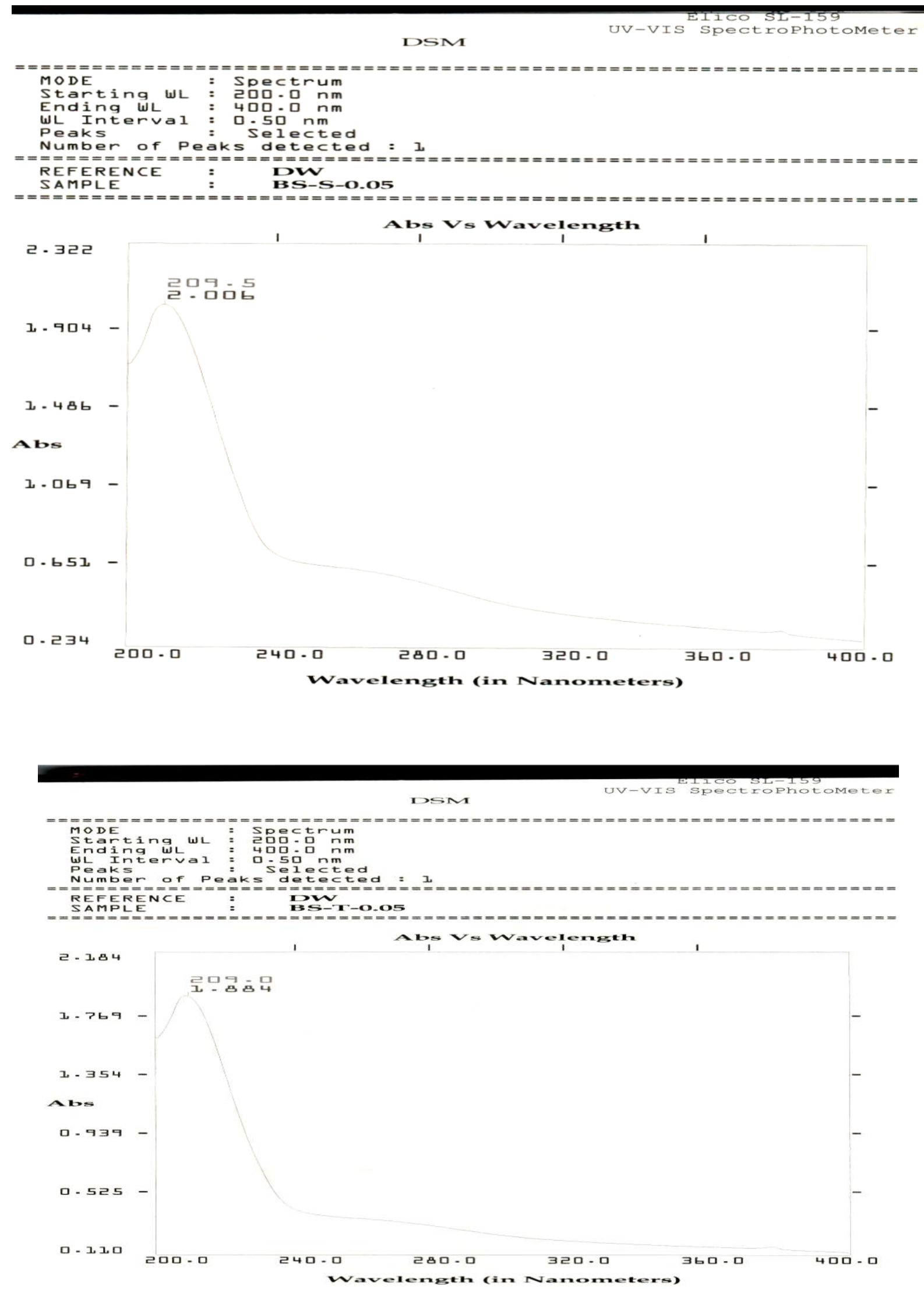

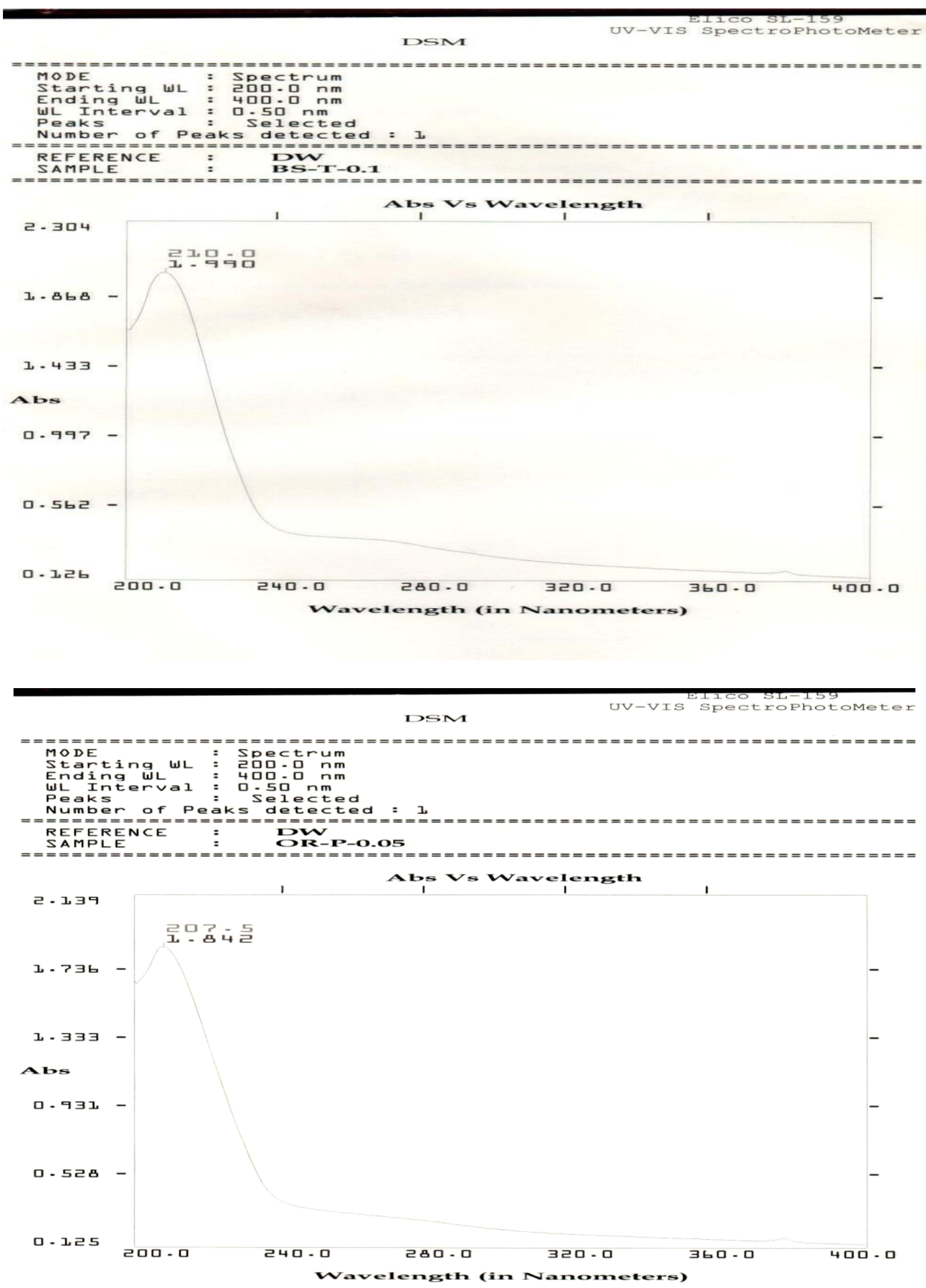

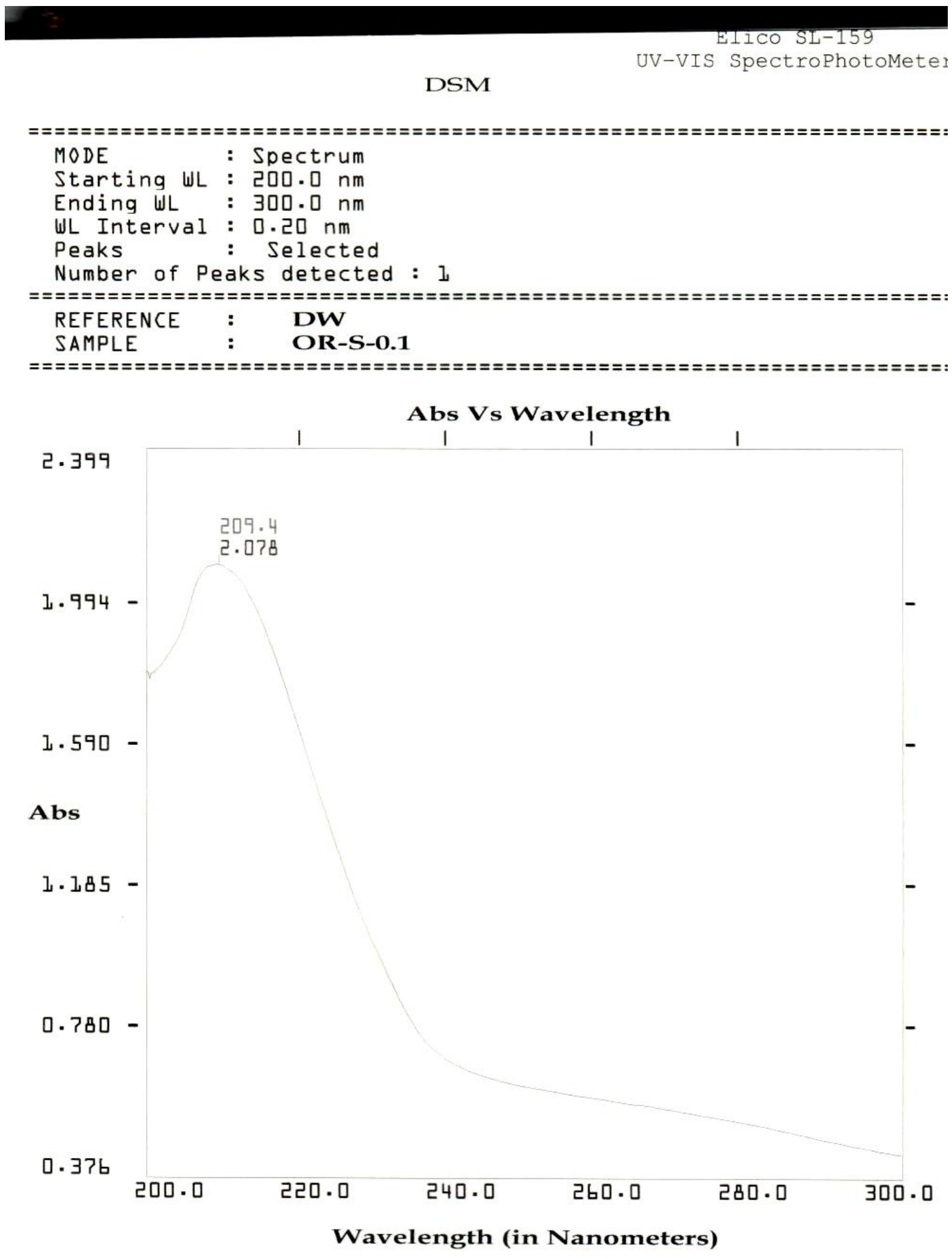

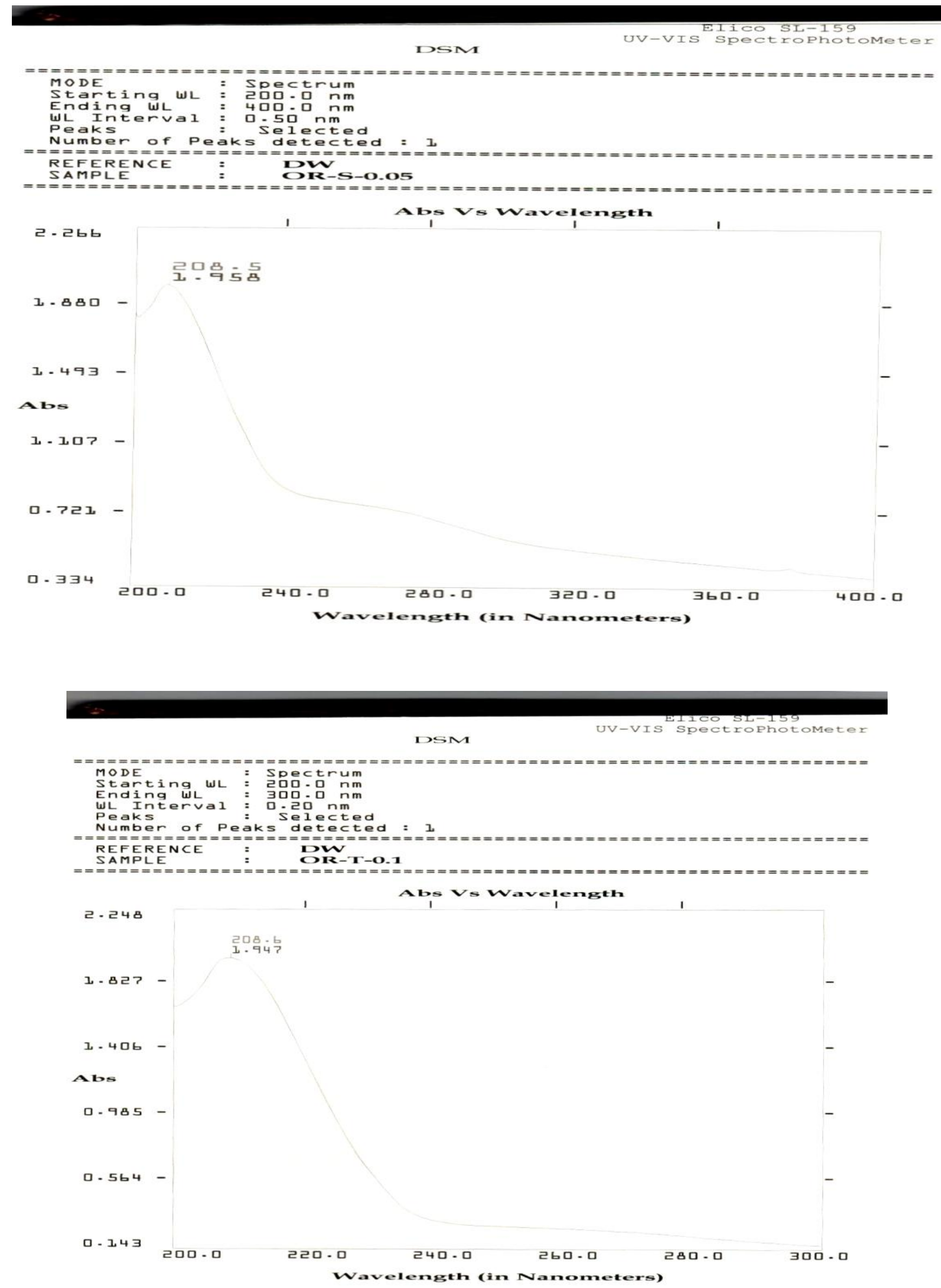


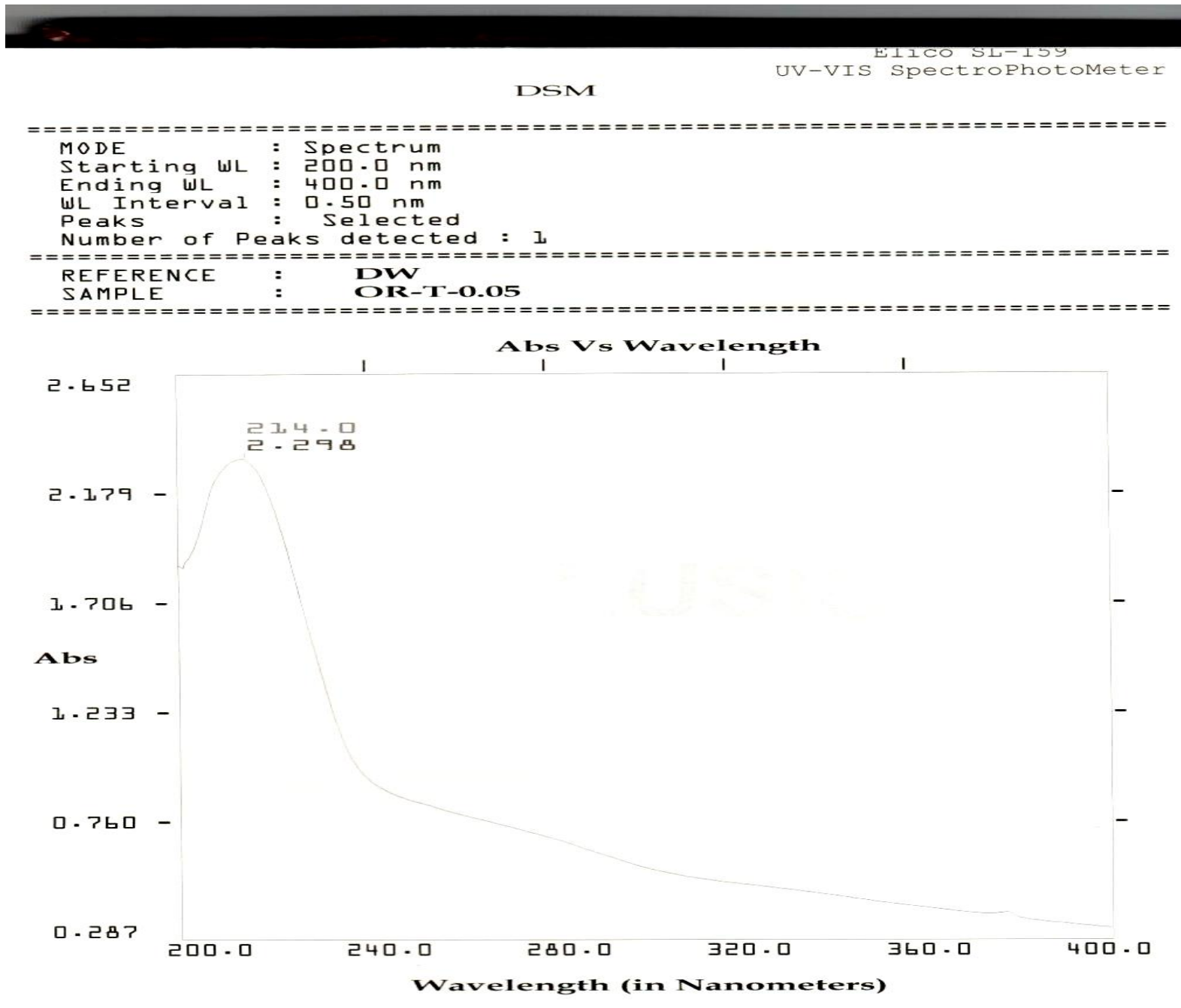

It has been emphasized by Toffes and Moseley (1953) that the production of fluorescent pigment by a strain of $\mathrm{P}$. aeruginosa is inversely related to the logarithm of the iron concentration of the medium. The presence of chelating agents can lower the concentration of iron. The metabolite iron is an absolute requirement for the synthesis of the cytochrome system enzymes.

The control of the availability of iron to bacteria using iron binding egg white protein conalbumin, appears to be an excellent method of assuring an efficient medium for use in the detection of fluorescent pigments.
When iron free conalbumin is present in the medium, the availability of iron is restricted.

With addition of $0.05 \%$ of phosphate in medium yellow pigmented colonies showed maximum pigmentation as compared to $0.1 \%$ of phosphate in the medium. No peaks were detected in presence of sulphate and tyrosine in the medium as shown in table 3.

In the presence of $0.05 \%$ of Phosphate in the medium the cream pigmented colonies show maximum pigmentation as compared to $0.1 \%$ of phosphate in the medium. These isolates also showed chromogenic characteristics in the presence of $0.1 \%$ and $0.05 \%$ of sulphate 
and tyrosine in the medium. The maximum pigmentation was seen in $0.1 \%$ sulphate and $0.1 \%$ tyrosine as shown in table 4 .

The maximum pigmentation of orange pigmented colonies was showed in $0.05 \%$ and no peak was detected in $0.1 \%$ phosphate in the medium. These pigmentation colonies also showed maximum pigmentation in the presence of other compounds such as sulphate and tyrosine with the concentration of $0.1 \%$ and $0.05 \%$ in the medium. The maximum pigmentation was seen in the medium containing $0.1 \%$ sulphate and $0.05 \%$ tyrosine as shown in the table 5 .

It is concluded that the increase in the concentration of Phosphate decrease the pigmentation characteristics of cream, orange and yellow pigmented bacteria.

High concentration of sulphate $0.1 \%$ and high concentration of tyrosine $0.1 \%$ enhances pigmentation production in cream pigmented bacteria whereas high concentration of sulphate $0.1 \%$ and low concentration of tyrosine $0.05 \%$ shows good pigmentation characteristics.

As pigments have been extensively evaluated over the years for its antibiotic, antigenic and antitumor effects, special consideration was made towards ability of chromogenic bacteria to produce pigmentation under certain chemical conditions. It was observed that the chemicals in study markedly affect the pigmentation characteristics of the mentioned isolates

\section{References}

Alport, D.C. and Bulloch. 1958. The pigmentation and cell wall material of Daldivia sp. J. Chem. Soc., 4090-4094

Aronson, J.N. and Vickers. 1965. Conversion of tyrosine to dihydroxy phenylalanine by a tyrosine hydroxylase from Bacillus cereus. Biochem. Biophys Acta, 110:624-626

Barnett, T. A and Hageman. 1983. Characterization of a brown pigment from Bacillus subtiliscultures. Can.J.Microbiol, 29:309-315

Burgova, M.P.; Loavyagina, E.V.; Falina, N.N. and Goldenberg, A.L. 1957. A study of the absorption spectra of the antibiotic bacterium Prodigiosum. Primenenie Metodov Spectroskipiiv Prom. Prodovol'stven, 1955:173-179

Buttiaux, R.; Beerens, H. and Tacquet, A. 1969. Manuel de techniques bacteriologiques. Ed.Med. Flammarion. Paris

Cruess-Callaghan, G. and Gorman, M.J. 1935. On the characteristics of Bacterium Violaceum (Schroter) and some allied species of violet bacteria. Sci.Proc.R.Dublin Soc., 21:213

Cuajolle, F.M.E.D.H.; Caujolle, S.B. Cross and Calver, M.M.Y. 1967. Limits of toxic and tetratogenic tolerance of dimethyl sulfoxide. Ann.N.Y.Sci., 141(1):100-126.

Dundas, I.D. 1977. Physiology of Halo bacteriaceae. Adv.Microb.Physiol., 15:85-120

Efimenko, O.M.; Koznetsova, G.A. and Yakimov, P.A. 1956. Prodigiosin-an antibiotic from bacterium Prodigiosum. Biokhimiya, 21(3):419-423

Eltinge, E.T. 1956. Nitrate reduction in the genus Chromobacterium. Antonie Van Leeuwenhoek. J.Microbiol.serol., 22:139-144

Flannery, W.L. 1956. Current status of knowledge of halophilic bacteria. Bacteriol. Rev., 20:49-66

Forsyth, M.P. and Kushner, D.J. 1970. Nutrition and distribution of salt response in populations of moderately halophilic bacteria. Can.J.Microbiol., $16: 253-261$ 
Gautheir, M.J Shewan, J.M.; Gibson, D.M. and Lee, I.V. 1975. Taxonomic position and seasonal variations in neritic environment of some gram-negative producing bacteria. J.Gen.Microbiol., 87:211-218

Gauthier, M.J. 1969. Substances antibacteriennes produites par les bacteries marines. I. Etude systematique de I'activite antagoniste de souches marins vis-à-vis germs telluriques aerobes. Rev.Int.Oceanogr.Med., 15-16:41-49

Gessard, C. 1892. Sur la function fluorescigene des microbes. Ann.Del'Inst.Pasteur, 6:801.

Gilman, J.P. 1953. Studies on certain species of bacteria assigned to the genus Chromobacterium. J.Bacteriol., 65:4852.

Greenberg, E.P. and Canale-Parola, E. 1976. Spirochaete halophila Sp.n., a facultative anaerobe from a high salinity pond.Archmicrobiol.,110:185-194

Grimont, P.A.D. and Grimont, F. 1984. Genus VIII. Serratia. In Bergey's manual of systematic bacteriology vol. I. Edited by N.R. Krieg Williams and Wilkins. Baltimore, pp 477-484

Hariman, B.K. and Udenfriend, S. 1969. A method for immediate visualization of proteins in acrylamide gels and its use for preparation of antibodies to enzymes. Anal.Biochem., 30:391-394

Hueppe, F. 1880. Etudes sur le lait blue. Cohn'sBeitrage zur Biologie de Pflanzen, 3.original not seen

Ivins, B.E. and Holmes, R.K. 1980. Isolation and characterization of melaninproducing (Mel) mutants of vibrio cholera. Infect Immun., 27:721-729

Jordon, E.O. 1899. Production of fluorescent pigment. Botanical Gaz., 27:19

Keradjopoulos, D. and Halldorf, A.W. 1977.Thermophilic character of enzymes from extreme halophilic bacteria. FEMS Microbiol. Lett., 1:179-
182.

Kraft, E. 1902. Beitrage zur biologie des B. prodigious and zum chemischen verhalten seines pigments. Thesis Wurzburg, Germany (Cited by Williams and Hearn. 1967).

Kushner, D.J. 1978. Life in high salt and solute concentrations: halophilic bacteria. In Microbial life in extreme environments, Edited by D.J. Kushner. AcademicPress, London. Pp. 318-358.

Kuster, E. 1976. Chromogenecity of actinomycetes. In Actinomycetes: The boundary microorganisms. Edited by $\mathrm{T}$. Arai, University Park Press, Tokyo. pp 43-53

Lanxyi, J.K. 1974. Salt dependent properties of proteins from Auremely halophilic bacteria. Bacteriol.Rev., 38:272-290

Larsen, H. 1962. Halophilism. In the bacteria.Vol. 4. Edited by I.C. Gunsalus and R.V. Stainer. Academic Press, New York, pp. 297-342

Larsen, H. 1967. Biochemical aspects of extreme halophilism. Adv Microb. Physiol, I:97-132

Leifson, E. 1956. Morphological and physiological characteristics of the genus Chromobacterium. J.Bacteriol., 71:393-400

Lepierre, C. 1895. Recherches sur la function fluorescigene d'un Bacille fluorescent pathogene. Ann.Del"Inst. Pasteur, 9:643.

Matheson, T.A., Sprott, G.G.'Mc Donald, I.J. and Tessier, H. 1976. Some properties of an unidentified halophile: growth characteristics, internal salt concentration, and morphology. Can.J. Microbiol., 22:780-786

Muller, H.E. 1985. Production of brownish pigment by bacteria of the Morganella Proteus Providencia group. Zentralbl. Bakteliol. Mikrobiol. Hyg.Abt.I, Orig.A, 240:428-435.

Paruchuri, D.K. and R.M. Harshey 1987. 
Flagellar variation in Serratia marcescens in associated with color variation. J. Bacteriol., 169:61-65

Pomerantz, S.H. and Murthy, V.V. 1974. Purification and properties of tyrosinases from Vibrio tyrosinasicus. Arch. Biochem. Biophys. 160:73-82

Sadasivan, L. and Neyra, C.A. 1987.Cyst production and brown pigment formation in ageing cultures of Azospirillum brasilense ATCC 29145. J. Bacteriol., 169:1670-1677.

Sneath, P.H.A. 1956a.Cultural and biochemical characteristics of the genus Chromobacterium. J.Gen.Microbiol., 15:70-98.

Stryer, I. 1965. The interaction of a naphthalene dye with apo myoglobin and apohemoglobin. J.Mol.Biol., 13:482-495.

Sullivan, M. 1905. Synthetic culture media and the biochemistry of bacterial pigments. J.Med.Res., 14:109.

Tanner, F.W. 1918. A study of green fluorescent bacteria from water. J.Bact., 3:63.

Vickers, R.M. and Yu, V.L. 1984. Clinical laboratory differentiation of legionellaceae family members with pigment production and florescence on media supplemented with aromatic substrates. J.Clin.Microbiol., 19:583587.

Vinas, M., Loren, J.G. and Guinea, J. 1983. Particulate bound pigment of Serratia marcescens and its association with cellular envelops. Microbios Lett., 24:19-26.

Weber, G. and Laurence, J.R. 1954. Fluorescent indicator of absorption in aqueous solution and on the solid phase. Biochem.J., 56: xxxi.

Wheeler, M.H. 1983. Comparision of fungal melanin biosynthesis in ascomycetous, imperfect and basidiomycetous fungi. Trans.Brit.Mycol.Soc., 81:29-36.

Yabuvchi, E. and Ohyama, A. 1972. Characterization of pyomelaninproducing strains of Pseudomonas aeruginosa. J. Syst.Bacteriol., 22:53-64. Yoshimoto, T., Yamamoto. K. and Tsuru, D. 1985. Extracellular tyrosinase from Streptomyces sp.KY 453: Purification and some enzymatic properties. J.Biochem., 97:1747-1754.

\section{How to cite this article:}

Mohammed Abdul Aleem Qureshi, Saravanan Sankaran and Sowrirajan Subbaiah. 2018. Effect of Certain Compounds on the Pigmentation Characteristis of Some Bacterial Isolates. Int.J.Curr.Microbiol.App.Sci. 7(01): 2464-2483. doi: https://doi.org/10.20546/ijcmas.2018.701.297 